\title{
CORRIGENDUM
}

\section{Distinct pattern of TP53 mutations in squamous cell carcinoma of the esophagus in Iran}

\author{
Alireza Sepehr, Philippe Tanière, Ghislaine Martel-Planche, Abed-Ali Zia'ee, \\ Ferdous Rastgar-Jazii, Mansour Yazdanbod, Gholamreza Etemad-Moghadam, \\ Farin Kamangar, Farrokh Saidi and Pierre Hainaut
}

DOI: $10.1038 / \mathrm{sj} /$ onc/1205254

Correction to: Oncogene (2002) 20, 7368-7374.

Since the publication of the above paper, the authors have identified an error in the abstract. A revised version of the abstract is given below.

Extremely high rates of squamous cell carcinoma of the esophagus (SCCE) are observed in Iran, reflecting unknown, genetic and/or epidemiological risk factors. Among genetic alterations in SCCE, TP53 mutations are the most frequent, vary among populations, and may provide clues on etiological mechanisms. We have analysed mutations in TP53 (exons 5-8) in 98 SCCE from Iran by temporal temperature gel electrophoresis and direct sequencing. We found 58 mutations in 49 patients $(50 \%)$, with a high prevalence of $\mathrm{C}$ to $\mathrm{T}$ transitions at CpG dinucleotides (29.3\%). The TP53 mutation pattern in Iran was significantly different from that observed in SCCEs from high incidence areas of China and Western Europe $(P=0.007)$. Moreover, the prevalence of mutations at $\mathrm{A}: \mathrm{T}$ base pairs (transitions and transversions) was higher in men than in women (38.7\% vs $11.1 \%, P=0.033)$. COX-2 overexpression was detected in $69 \%$ of the cases evaluated $(24 / 35)$, without significant association with TP53 mutation. Accumulation of nitrotyrosine, a marker of protein damage by excess levels of nitric oxide, was observed in tumor cells in six of 18 cases analysed. These results are consistent with the hypothesis that several factors are involved in TP53 mutagenesis in Iran. These factors include a baseline of chronic inflammatory stress, which may have a multiplicative impact on the sensitivity of esophageal cells to exogenous factors of risk. 